\title{
ROD-LOADED AND PBG MULTI-BEAM KLYSTRON CAVITIES *
}

\author{
A. V. Smirnov, D. Yu, DULY Research Inc., Rancho Palos Verdes, CA 90275, U.S.A.
}

\section{Abstract}

Rod-loaded structures were studied for multi-beam and single-beam metal cavities. Coupling efficiency, parasitic modes and their dependence on inter-beam spacing for a 6-beam, high-power, X-band MBK were included in this study. Realization of Photonic Band Gap (PBG) effect in klystrons was also considered.

\section{INTRODUCTION}

Lattice-like structures with transverse periodicity, such as rod-loaded and PBG cavities and waveguides, have promising applications in both particle acceleration and $\mathrm{rf}$ generation $[1,2,3]$. For a single-defect structure, a strong effect of HOM Q-suppression was revealed; for a 6-defect cavity, methods for effective excitation and outcoupling of the trapped $\mathrm{TM}_{010}$ mode were developed [1]. Advanced flat-field rod-loaded structures for sheet beam klystron and lasertron were also proposed $[2,3]$.

Earlier a magnetron injection gun (MIG) was proposed for an X-band cluster klystron [4] to deliver $26 \mathrm{MW} /$ beam $\mathrm{RF}$ power at $36 \mathrm{~A} / \mathrm{cm}^{2}$ cathode loading. Compared with Pierce guns, the MIG has an advantage of as high as 40$50 \mathrm{~A} / \mathrm{cm}^{2}$ emission [5,6], up to 100,000 hours lifetime [7], and reduced spacing between adjacent beamlets $(\sim 2.4-$ $2.6 \mathrm{~cm}$ at $11.4 \mathrm{GHz}$ ). In spite of inherent beam noise and velocity spread, such guns may be effective for future high-frequency multi-beam klystrons.

We consider rod-loaded multi-beam cavity designs numerically with special attention paid to coupling efficiency, inter-beam spacing, and parasitic modes.

\section{6-DEFECT CAVITIES WITH CENTRAL COUPLING}

Here we focus primarily in a 6-beam output cavity with central coupling to operate at $\mathrm{f}=11.4 \mathrm{GHz}$ and inter-beam spacing $\mathrm{R}_{\text {def }} \geq 2.5 \mathrm{~cm}$ (Fig. 1). An initial $\mathrm{TM}_{010}$ cavity design with a circular $\mathrm{TM}_{01}$ output waveguide demonstrated good efficiency [3], but insufficient frequency separation $\Delta \mathrm{f} / \mathrm{f}$ between the fundamental and closest parasitic (dipole in this case) mode, low spacing between beamlet centers $\mathrm{R}_{\text {def }}$, and too high external Qfactor $\mathrm{Q}_{\text {ext }}$ (see Table 1, variant \#5).

Table 1 presents the results for trapped (defect) modes (lines \#1-5), where $b$ is lattice spacing, a is the rod radius, and $R_{\text {pipe }}$ is the radius of the circular waveguide. The last line corresponds to a global $\mathrm{TM}_{030}$ mode having insignificant trapping into the defects. The closest parasitic modes are dipole $(\# 1,2,5)$, quadrupole-like $(\# 3,6)$, and sextupole, localized in the defects (\#4).

In spite of an oversized pipe, Variants \#1,2 are undercoupled. The defects are separated by several lattice cells with relatively thick rods (compared to [1]) resulting

* Work supported by DOE SBIR grant no. DE-FG02-03ER83845 in a dense modal spectrum. An overmoded waveguide would introduce additional modal hybridization.

Table 1: Parameters of 6-defect, rod-loaded cavity designs with central waveguide outcoupling at $11.4 \mathrm{GHz}$.

\begin{tabular}{|c|c|c|c|c|c|c|c|c|}
\hline \# & $\frac{0}{\frac{0}{0}}$ & 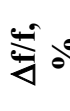 & 今 & $\hat{F}$ & 竎 & 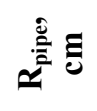 & $\begin{array}{l}\Xi \\
\text { ¿ } \\
0\end{array}$ & a \\
\hline 1 & $\mathrm{TM}_{030}$ & 0.2 & $>2 \cdot 10^{4}$ & 0.15 & 4.2 & 1.81 & 0.81 & \\
\hline 2 & $\mathrm{TM}_{020}$ & 0.8 & $>2 \cdot 10^{2}$ & 0.15 & 2.9 & 2.02 & 0.82 & \\
\hline 3 & $\mathrm{TM}_{030}$ & 0.4 & 280 & 0.066 & 3.6 & 1.17 & 0.7 & \\
\hline 4 & $\mathrm{TM}_{0}$ & 0.9 & 78.5 & 0.066 & 2.5 & 1.21 & 0.72 & 22.2 \\
\hline 5 & $\mathrm{TM}_{0}$ & 0.1 & 350 & 0.12 & 2.1 & 1.04 & 1.01 & 16 \\
\hline 6 & $\mathrm{TM}_{030}$ & $>5$ & $\leq 60$ & 0.066 & 3 & 1.21 & 0.86 & 6.54 \\
\hline
\end{tabular}

Thinner rods and/or smaller inter-defect spacing increase the coupling and frequency separation for lowest trapped modes (variants \#1-5). It is a result of increased defect-to-defect coupling [2,3] and a decrease in occupied volume (area). Qualitatively the last effect can be evaluated with the scaling of eigenmode spectrum density $\Delta \mathrm{N} / \Delta \omega$ given by $[8]$ :

$$
\left.\frac{\Delta N}{\Delta \omega}\right|_{3 D} \approx \frac{V \omega^{2}}{2 \pi^{2} c^{3}},\left.\quad \frac{\Delta N}{\Delta \omega}\right|_{2 D} \approx \frac{S \omega}{\pi c^{2}},
$$

where $\mathrm{V}$ and $\mathrm{S}$ are the cavity volume and area, and $\mathrm{N}$ is the eigenmode number.

Variant \#4 (see Fig. 1) is preferable among \#1-5 in terms of shunt impedance and outcoupling for the operating mode. $\mathrm{R} / \mathrm{Q}$ and $\mathrm{Q}_{\mathrm{ext}}$ are close to that found to be optimal for a $500 \mathrm{kV}, 130-150 \mathrm{MW}$ design [1]. The closest unwanted mode is a higher-frequency, trapped sextupole mode, which is anti-phased for adjacent defects. Another side mode is a $\mathrm{TM}_{220}$-like quadrupole mode downshifted by $1.04 \%$. The frequency separation remains low: $\Delta \mathrm{f} / \mathrm{f}<1 / \mathrm{Q}_{\text {loaded }}$, where the figure of merit $\mathrm{Q}_{\text {loaded }}$ includes beam loading. Mode competition and parasitic coupling can be reduced with decoupling of idle cavities, each of them to be individual per beam.

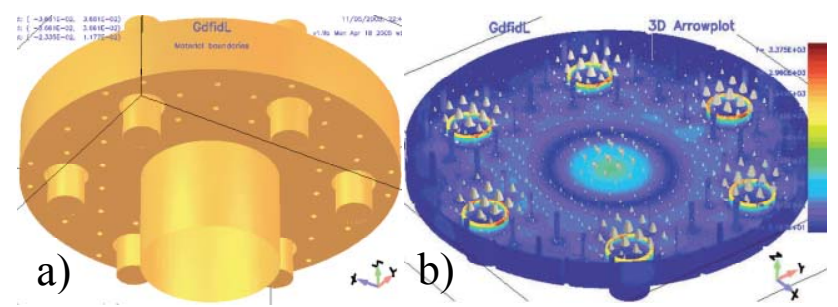

Figure 1: (a) 6-defect cavity with central pipe, (b) the lowest trapped operating mode for Variant \#4 in Table 1.

Another common problem of the lowest $\mathrm{TM}_{\mathrm{ON} 0}$ modes trapped into the defects is that the field amplitudes calculated for different defects do not coincide. This phenomenon was noticed in a previous design for the 
$\mathrm{TM}_{010}$ operating mode (including 2D SUPERFISH simulations with triangular mesh). The relative difference of the impedances between defects may vary from 3-4\% to tens of percentages dependently on $\mathrm{a} / \mathrm{b}$ ratio, lattice configuration, defect topology, pipe length, and basic mesh step (which was always less than the rod radius). Review of the results indicates that other monopole modes with less concentration in the defects (global modes $\mathrm{TM}_{010}$ and $\mathrm{TM}_{030}$ ) do not have such a big inhomogeneity, even for a courser mesh and higher frequencies $\left(\mathrm{TM}_{030}\right.$ mode). Hybridization may also contribute to this effect by coupling with one of the quadrupole modes having less relative frequency separation compared to other modes (non-defect $\mathrm{TM}_{010}$ and $\mathrm{TM}_{030}$ in the $\# 2,4,6$ variants).

Though the $\mathrm{TM}_{030}$ mode of Variant \#6 is weakly trapped into the defects (see Fig. 2), it can still be considered as a candidate for operating mode due to the larger frequency separation, much better field uniformity and bigger spacing between beamlets $\left(\mathrm{R}_{\mathrm{def}}=3 \mathrm{~cm}\right)$. The shunt impedance is low $(\mathrm{R} / \mathrm{Q}=6.54 \Omega)$, but it is close to that for a HOM ring cavity with $\mathrm{R} / \mathrm{Q} \approx 7 \Omega$ in a $150 \mathrm{MW}, 6$ beam, X-band klystron design [9]. The calculated frequency separation is $10.5 \%$ from the closest $\mathrm{TM}_{220}$-like mode. Lower shunt impedance means higher external Qfactor $\left(\mathrm{Q}_{\mathrm{ext}}>100\right)$ for klystron parameters of interest $[1,9]$. Higher external Q-factor simplifies the coupler design and eases requirements on frequency separation. For this design we can achieve $\Delta \mathrm{f} / \mathrm{f} \gg>1 / \mathrm{Q}_{\text {loaded }}$, making it more stable to parasitic mode interactions and beam-beam oscillations.

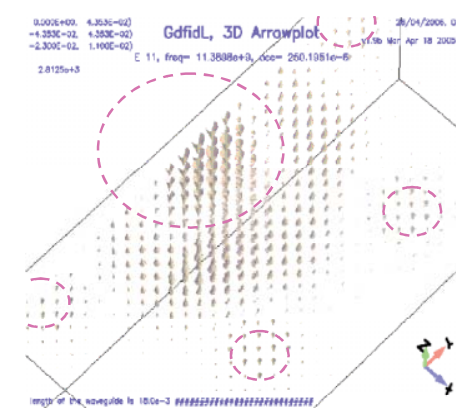

Figure 2: $\mathrm{TM}_{030}$ mode in a half-cavity strongly coupled with a central pipe (variant \#6 of Table 1). Material boundaries (not shown) are similar to that in Fig. 1 . Dashed lines denote the $\mathrm{TM}_{01}$ pipe and the beamlets.

\section{6-DEFECT OUTPUT CAVITY WITH SIDE OUTCOUPLING}

The design shown in Fig. $3 \mathrm{~b}$ can provide high efficiency and power $(\sim 63 \%, 150 \mathrm{MW}$ in the $\mathrm{X}$-band [1]). It has two side ports with high $\mathrm{R} / \mathrm{Q}=\mathrm{U}^{2} / 2 \omega \mathrm{W}=23 \Omega$ per beamlet and low enough $\mathrm{Q}_{\mathrm{ext}} \approx 50$. Optimization for $\mathrm{TM}_{010}$ trapped mode yielded a closest frequency separation of about 5-6\%. Scaled to L-band, this design is quite comparable with ring cavities: its diameter is intermediate between VKL 8301 CPI [10] $\varnothing \sim 48 \mathrm{~cm}$ and $\varnothing \sim 24 \mathrm{~cm}$ Toshiba E-3736 [11] designs, and provides a higher R/Q (by 20-30\%). However, at X-band the dimensions are very small: rod diameter $\varnothing \approx 1 \mathrm{~mm}$ and distance between beamlets $\mathrm{R}_{\mathrm{def}} \approx 1.1 \mathrm{~cm}$. It requires additional $R \& D$ efforts to develop a double-convergent or special gun having a large common cathode and a system of anodes/grids to form six good-quality beams. Field homogeneity between beamlets can be influenced by quadrupole-like asymmetry induced by side outcoupling.
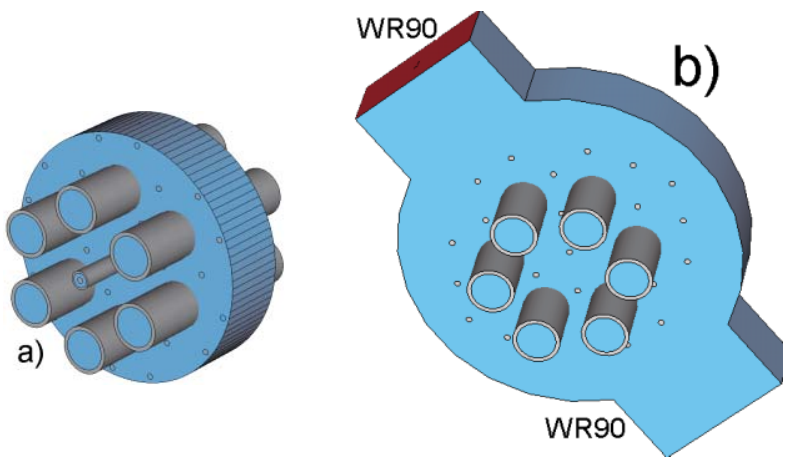

Figure 3: $\mathrm{TM}_{010}$ rod-loaded cavities for a compact 6-beam klystron: (a) input cavity with coaxial central-rod coupling, and (b) output 2-port side coupling cavity.

The multi-defect structures described above allow a sufficient outcoupling, but at high frequencies the design becomes a very tight compromise between inter-beam spacing, $\mathrm{R} / \mathrm{Q}, \mathrm{Q}_{\text {ext }}$, and frequency separation. For a 6defect cavity the "normal" modes are represented by a monopole, 2 dipole, 2 quadrupole, and a sextupole modes $[1,2,3]$. For an output cavity we may have a $\mathrm{Q}_{\text {loaded }}$ for fundamental mode much smaller than that for side modes. Introduction of absorber or side opening into the multidefect structures does not discriminate unwanted modes. The defects act as a whole group (a cluster) of dominant (trapped) modes having close frequencies and Q-factors.

\section{TOWARDS A PBG KLYSTRON}

The key parameter for practical applications of the PBG effect is Q-factor reduction for HOMs with respect to that for fundamental mode. One can model this effect numerically for a realistic cavity using an eigenmode approach in a cavity having no ports but terminated with a periphery absorber or a lossy metal. The replacement of the periphery absorber by a highly lossy metal does not affect significantly the eigenmode structure due to oversized (for $2^{\text {nd }}$ and higher order) lattices. In Fig. 4 we plotted the Q-factor ratio for damped and all-copper cavities for fundamental mode $\mathrm{Q} / \mathrm{Q}_{\mathrm{Cu}}$, for dipole mode $\mathrm{Q}_{110} / \mathrm{Q}_{110 \mathrm{Cu}}$, and the frequency ratio between the two modes $\mathrm{f}_{110} / \mathrm{f}_{010}$ for a triangular, 36-rod lattice with variable spacing $b$. One can see that in the operating region $0.139<\mathrm{a} / \mathrm{b}<0.184$ the dipole mode $\mathrm{Q}$ is damped by a factor of $\sim 30$, whereas the fundamental mode Q-factor reduction is insignificant $(<10 \%)$. The forbidden gap interleaves between the points of strong bifurcation: $a / b=0.19$ and 0.3 . Brillouin diagram for higher frequencies was obtained also with this frequency-domain method. The results show that other HOMs are damped as well in the allowed zone (by a factor of $>25,[1]$ ). Usage of MF112 
Eccosorb $^{\mathrm{TM}}$ at the periphery drops the Q-value of the dipole mode by a factor of $\sim 18-20\left(\mathrm{Q}_{110} \sim 300\right)$, whereas the fundamental mode $\mathrm{Q}-$ factor is reduced by $\sim 1.5 \%$ only. Matching of absorber material and shape optimization may improve these parameters further. These heavily damped PBG structures can be used as klystron gain cavities.

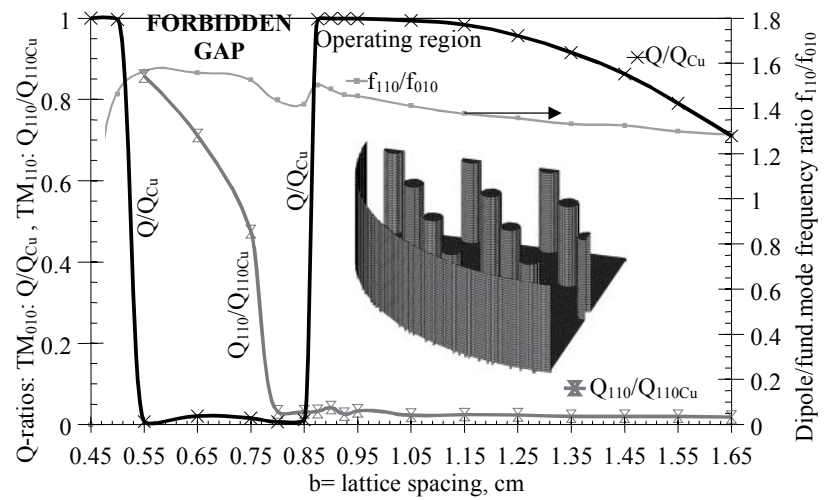

Figure 4: Q-factor reduction for a damped 36-rod PBG cavity for $\mathrm{TM}_{010}$ and $\mathrm{TM}_{110}$ modes. $\mathrm{a}=1.6 \mathrm{~mm}$, longitudinal gap $\mathrm{d}=6.91 \mathrm{~mm}$, periphery wall conductivity $\sigma_{\mathrm{w}}$ is assumed $10^{-8}$ of that for copper $\sigma_{\mathrm{Cu}}$.

The next step is to design structures strongly coupled for fundamental mode, but still damped for HOMs. For side-coupler geometry it can by done with insertion of one or two rectangular waveguides as close to the defect as possible (see Fig. 5).

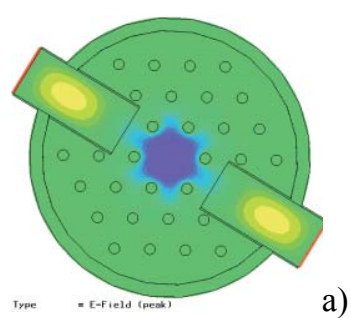

a)

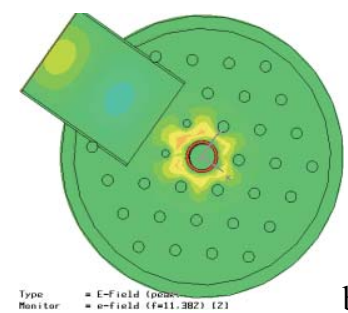

b)
Figure 5: (a) 2-port "pillbox" and (b) single-port "reentrant" X-band PBG cavity coupled to the $\mathrm{TM}_{010}$ mode only. $\mathrm{d}=7.9 \mathrm{~mm}$, absorber thickness $\Delta \mathrm{r}=3.8 \mathrm{~mm}$.

To provide $\mathrm{Q}_{\mathrm{ext}}-50-100$ for the single-port design (Fig 5(b)) as low as that for the 2-port design (Fig. 5(a)) we widened the waveguide and reduced the radius of two rods facing the waveguide (b): $a / b=0.1$ instead of regular $\mathrm{a} / \mathrm{b}=0.15$. The reduction of unloaded $\mathrm{Q}$-factor for $\mathrm{TM}_{010}$ mode and matched absorber is $\sim 4 \%$. Presence of the coupler does not eliminate the PBG effect: even with two waveguides (see Fig. 6) one can obtain $Q_{110} \sim 130$, $\mathrm{Q}_{120} \sim 80-57$. Such a heavy damping is quite comparable with the Manifold Damping Detuned Structures (DDS) where minimal Q-factors achievable for lower dipole band are as low as 300-10 [12].

An X-band, 6-beam PBG klystron can be designed as a modular combination of individual PBG klystrinos with three 3-port (or two 4-port) external combiners $[4,6]$.

Thus PBG-based solutions applied to higher frequency klystrons are capable of bringing about a number of benefits: discriminative suppression of all HOMs for all cavities with frequency separation $>30 \%$, sufficient cathode area suitable for MIG or conventional gun injection; sufficiently low external Q-factor along with high $\mathrm{R} / \mathrm{Q}$ for the operating mode; inter-beam uniformity; and relatively thick rods. PBG-based design is also beneficial for distributed multi-cavity power extraction due to inherent HOM suppression. Finally, another type of coupler, e.g., a 4-rod outcoupler with suppressed transformation into parasitic modes [13], could also be effective for multi-cell PBG structures, including accelerators.
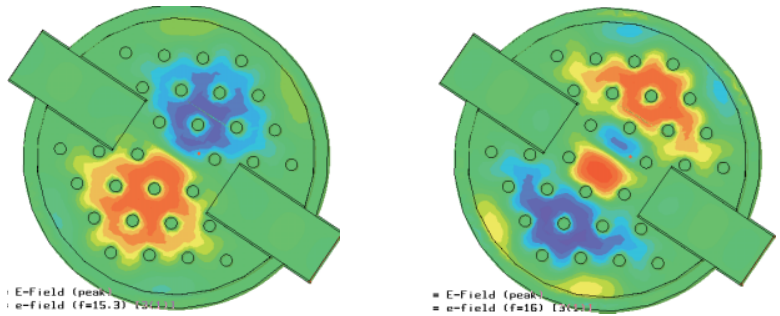

Figure 6: $\mathrm{TM}_{110}$ and $\mathrm{TM}_{120}$ modes excited with a current shifted asymmetrically from the defect center and at $45^{\circ}$ with respect to the waveguide axis.

\section{REFERENCES}

[1] A.V. Smirnov, D. Yu, in AIP Conf. Proc., v. 737 (2004) 722.

[2] D. Yu, D. Newsham, A. Smirnov, Proceedings of AAC02, AIP 647, 2002, p. 394.

[3] D. Yu, D. Newsham, A. Smirnov, Proceedings of PAC03, p. 1153.

[4] DOE SBIR Phase II Final Report. Grant \# DE FG03-93ER81573, DULY Research Inc. (1997).

[5] H. Wang, R. Palmer, J. Gallardo, in Proc. of Int. Workshop on Pulsed RF Power for Liner Colliders (RF-93), Dubna, Russia (1993).

[6] R.B. Palmer, W.B. Herrmannesfeld, K.R. Eppley, in Particle Accelerators, v.30, No1-4 (1990) 197.

[7] S. A. Cherenshchikov, A. N. Dovbnya, A. N. Opanasenko, in Proc. PAC95 (1995) 938.

[8] R. Courant, D. Gilbert. Methods of Mathematical Physics, V. 1, Gosteckhizdat, 1933 (in Russian).

[9] A. Larionov, S. Matsumoto, V. Teryaev, in Proc. of 9th. Int. Workshop on Linear Colliders, LC2002, Febr. 4-8, 2002, SLAC, Stanford, California.

[10]E. Wright, A. Balkcum, H. Bohlen et al., in Proc. of EPAC2004, Lucerne, Switzerland (2004) 1117.

[11] Y.H. Chin, Toshiba/KEK MBK for TESLA: Design, Status and Schedule. 2004/1/26, Tesla Collaboration Meeting. DESY, Berlin, January, 21-23 (2004).

[12] R.M. Jones, N.M. Kroll, T. Higo, R.H. Miller, R.D Ruth, PAC2001 Conf. Proc., Chicago, IL (2001) 936.

[13] A.V. Smirnov, D. Yu, "Ceramic Power Extractor Design for $15.6 \mathrm{GHz}$," these Proceedings. 